\title{
Exfoliation of hematite: Morphological, structural and magnetic investigations.
}

\author{
Anup Kumar, Rui Zhang, M. Venkatesan, Plamen Stamenov and J. M. D. Coey* \\ School of Physics, Trinity College, Dublin 2, Ireland
}

\begin{abstract}
Three natural specimens of hematite are characterized using X-ray diffraction, magnetometry IR/Raman and Mössbauer spectroscopy, with focus on the Morin transition. All are exfoliated by grinding and prolonged sonication in dimethylformamide, followed by centrifuging to extract exfoliated debris from the supernatant. This material is characterized by electron microscopy and atomic force microscopy. There are three main $\mathrm{Fe}_{2} \mathrm{O}_{3}$ constituents: lamellae that are hundreds of nanometers in diameter and typically $1-10 \mathrm{~nm}$ thick, nanoparticles that are about $100 \mathrm{~nm}$ in size and ultra-fine nanoparticles smaller than $10 \mathrm{~nm}$. Estimated yield of the lamellae is $10^{-3}-10^{-4}$ by volume. All the iron in the debris is antiferromagnetically ordered at room temperature, including $30 \%$ that is superparamagnetic and contributes to a quadrupole doublet in the Mössbauer spectrum, but the characteristic weak net moment due to spin canting is absent. Unlike the normal hematite nanoparticles, there is no Morin transition and no net moment in the range $100-300 \mathrm{~K}$. The absence of a net moment is understandable because the lamellae cleave in planes that make small angles with the $c$-axis
\end{abstract}

Keywords: hematite, liquid-phase exfoliation, 2D oxide materials, ultra-thin antiferromagnetic oxides, hematene, ferric oxide nanoparticles

*Corresponding author's electronic mail: jcoey@tcd.ie 


\section{Introduction}

Hematite $\left(\alpha-\mathrm{Fe}_{2} \mathrm{O}_{3}\right)$ is the major iron rock-forming mineral and, along with magnetite $\left(\mathrm{Fe}_{3} \mathrm{O}_{4}\right)$, it is one of two archetypical naturally-occurring magnetic materials. Like magnetite, hematite has played a significant part in developing key concepts in localized magnetism such as antiferromagnetic spin-reorientation transitions, the Dzyalsohinskii-Moriya interaction, magnetocrystalline anisotropy of S-state ions [1] and recently, topologically-protected antiferromagnetic vortex excitations [2]. Both hematite and magnetite are crystals with threedimensional crystal structures, where the bonding is principally ionic.

Recently, Balan et. al., reported that natural mineral specimens can be ultrasonically exfoliated in a solvent to yield ultra-thin sheets that they called 'hematene' [3] or 'magnetene' [4]. Other authors previously synthesised magnetic half-unit-cell thick $\alpha-\mathrm{Fe}_{2} \mathrm{O}_{3}$ nanosheets from a goethite precursor, using template-assisted oriented growth [5]. Exfoliation is often possible in two-dimensional layered materials such as clay minerals, transition metal trihalides or graphite, where covalent or ionic bonding within layers is strong, but the interlayer bonding is much weaker and has van der Waals character. It may then be possible to separate the layers mechanically, or chemically by exfoliating with an appropriate solvent. Alternatively, twodimensional sheets of transition metal compounds may be intercalated in graphite. There has been a recent revival of interest in these systems as two-dimensional magnetic model compounds and as possible components of device hererostructures [6,7]. The results of Balan et al suggest that exfoliation may be extended beyond traditional 2D materials with van der Waals interlayer bonding.

Hematite crystallizes in the corundum structure with rhombohedral space group $\mathrm{R} \overline{3} \mathrm{c}$, but the structure is usually indexed on a hexagonal cell with $a=503.6 \mathrm{pm}, c=1375.3 \mathrm{pm}$. It is perhaps the best-known antiferromagnet, with a Néel temperature $T_{\mathrm{N}}$ of $955 \mathrm{~K}$ due to strong $\mathrm{Fe}^{3+}-\mathrm{Fe}^{3+}$ interlayer superexchange. The antiferromagnetic axis lies at about $10^{\circ}$ to the $c$-axis at temperatures below the Morin transition at $T_{\mathrm{M}} \approx 260 \mathrm{~K}$ [8]. At higher temperatures, the moments lie in the basal $c$-plane, where they cant (tilt slightly) under the influence of the Dzyaloshinskii-Moriya interaction D. $\left(\mathbf{S}_{\mathrm{i}} \times \mathbf{S}_{\mathrm{j}}\right)$ to develop a weak net in-plane ferromagnetic moment. The net magnetization of the oxide at room temperature is usually about $0.3 \mathrm{Am}^{2} \mathrm{~kg}^{-1}$ or $0.003 \mu_{\mathrm{B}} / \mathrm{Fe}^{3+}$ ion. 
Here we characterize three natural hematite specimens and investigate the magnetic properties and structure of the products of prolonged sonication, following the procedure described in [3].

\section{Materials and Methods}

The three specimens are from different sources in England, USA and Italy [9]. Their provenance and other details are listed in Table 1. The Cumbria hematite H1 included singlecrystal platelets $2-3 \mathrm{~mm}$ in diameter in association with quartz, whereas the Utah hematite $\mathrm{H} 2$ was a lamellar specularite and the Italian specimen $\mathrm{H} 3$ was a mass of intergrown hematite crystals.

$50 \mathrm{mg}$ samples of hematite from each provenance were ground to a fine powder with mortar and pestle for X-ray, magnetic, optical and Mössbauer analysis. X-ray diffraction (XRD) patterns recorded on a Panalytical X'Pert Pro diffractometer with filtered $\mathrm{CuK}_{\alpha}$ radiation $(\lambda=154.18 \mathrm{pm})$ were refined using FULLPROF software. Magnetic analysis was performed using a Quantum Design MPMS XL5 SQUID magnetometer on powders contained in gelcap sample holders mounted in plastic straws. All isothermal magnetization curves have been corrected for the linear diamagnetic response of gelcap.

Further $50 \mathrm{mg}$ samples of the three materials were ground to fine powder and dispersed in $200 \mathrm{ml}$ of anhydrous dimethylformamide (DMF). The suspensions were sonicated for 72 hours in an ultrasonic bath. The resulting reddish-orange solution was then centrifugated at 5,000 rpm and the upper part of the supernatant solution was filtered to yield about $1 \mathrm{mg}$ of material on a Whatman filtration membrane with a pore size of $0.22 \mu \mathrm{m}$. The phase purity and crystallinity of both the original hematite and the filtrate were investigated by XRD. Morphological analysis was carried out on a Zeiss ULTRA plus scanning electron microscope (SEM). A JEOL 2100 $\mathrm{LaB}_{6}$ microscope operating at 100-200 $\mathrm{kV}$ was used for transmission electron microscopy (TEM) and an FEI Titan microscope was used for high resolution TEM (HRTEM) and selected area electron diffraction (SAED). For atomic force microscopy, a Digital Instruments Nanoscope 3 AFM was used in tapping mode. The hematite powder was mounted on Si (100) wafers for AFM imaging. SEM and TEM analysis of exfoliated hematite were conducted on samples obtained by evaporating supernatant solution, drop cast onto copper grids. Raman measurements were carried out in a Horiba Raman microscope with a 50× objective using a $\mathrm{He}-\mathrm{Ne}$ laser of 
wavelength $632.8 \mathrm{~nm}$ and infrared spectroscopy was performed on samples on filter paper using a Perkin Elmer Spectrum 100 FTIR/ATR. The ${ }^{57}$ Fe Mössbauer spectra were collected at room temperature in constant acceleration mode in transmission geometry with a ${ }^{57} \mathrm{Co}(\mathrm{Rh})$ source. Exfoliated hematite absorbers were dry, folded Whatman filter paper. Magnetization was measured on the SQUID magnetometer using $4 \times 5(\mathrm{~mm})^{2}$ pieces of filter paper mounted in a plastic straw. Data were corrected for the diamagnetism of the paper.

\section{Results}

Figure 1a shows a typical hematite XRD patterns for samples H1 and H2. Lattice parameters are included in Table 1. Both hematite samples were associated with minor amounts of quartz. No significant differences were found in the lattice parameters of any of the three hematite samples.
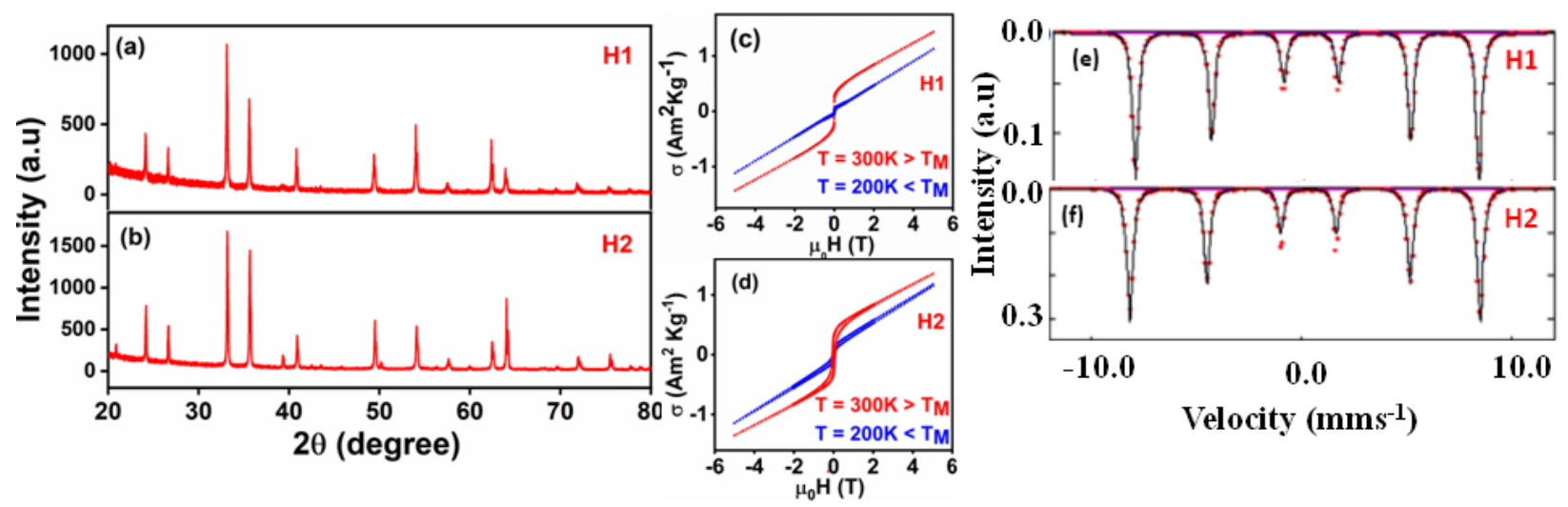

Figure 1 (a) and (b) XRD patterns for pure hematite samples H1 and H2; (c) Magnetization data for $\mathrm{H} 1$ at temperatures above $T_{\mathrm{M}}$ (red) and below $T_{\mathrm{M}}$ (blue); (d) Magnetization data for $\mathrm{H} 2$ at temperatures above $T_{\mathrm{M}}$ (red) and below $T_{\mathrm{M}}$ (blue); (e) and (f) Room temperature ${ }^{57} \mathrm{Fe}$ Mössbauer spectra with least-squares fits for sample $\mathrm{H} 1$ and $\mathrm{H} 2$.

The magnetic properties of hematite are evidently dependent on temperature, with a clear difference between the magnetization curves at $200 \mathrm{~K}$ and $300 \mathrm{~K}$ (Figure 1c and d), in agreement with much previous literature $[1,3,4]$. The measured saturation magnetization $\sigma_{\mathrm{s}}$ is $0.36-0.42$ $\mathrm{Am}^{2} \mathrm{~kg}^{-1}$ at $300 \mathrm{~K}$, with a dimensionless susceptibility (obtained from the high-field slope, taking the density as $5150 \mathrm{~kg} \mathrm{~m}^{-3}$ ) of $1.15-1.52 \times 10^{-3}$, consistent with the high Néel temperature. The Morin transition onset was found lie in range $245-251 \mathrm{~K}$. The transition extends over about 15 
$\mathrm{K}$ in each case. Coercivity is quite variable because it depends on crystallite size and it ranges 4$80 \mathrm{mT}$.

Mössbauer spectra for samples $\mathrm{H} 1$ and $\mathrm{H} 2$ exhibit the characteristic $\mathrm{Fe}^{3+}$ hematite sextet with hyperfine field $B_{\mathrm{hf}}=51.2-51.8 \mathrm{~T}$, isomer shift relative to iron IS $=0.41-0.44 \mathrm{mms}^{-1}$ and quadrupole shift $-0.03 \mathrm{mms}^{-1}$ (Figure 1e and f). Data on both natural hematite samples are summarized in Table 1, where they are compared with data on pure synthetic reference material taken from References 8 and 10.

Table 1: Summary of structural and magnetization data for three natural hematites and synthetic reference material.

\begin{tabular}{|c|c|c|c|c|c|}
\hline Sample & Provenance & Composition & $\begin{array}{l}\text { Lattice } \\
\text { parameters } \\
a, c(p m)\end{array}$ & $\begin{array}{l}{ }^{s}\left(\mathrm{Am}^{2} \mathrm{~kg}^{-1}\right) ; \\
\mu_{0} \mathrm{H}_{\mathrm{c}}(\mathrm{m} \mathrm{T}) ; \\
\chi\left(10^{-3}\right) ; T_{M}(\mathrm{~K})\end{array}$ & $\begin{array}{l}\text { Mössbauer } \\
\text { IS }\left(\mathrm{m} \mathrm{m} \mathrm{s}^{-1}\right) ; \\
\qquad / \mathrm{QS}\left(\mathrm{m} \mathrm{m} \mathrm{s}^{-1}\right) ; B_{\mathrm{hf}}(\mathrm{T})\end{array}$ \\
\hline H1 & Cumbria, UK & $\begin{array}{l}\text { Hematite } \\
\text { Quartz }\end{array}$ & $\begin{array}{l}a=504.1 ; c=1376.9 \\
a=491.4 ; c=540.4\end{array}$ & $0.36 ; 4 ; 1.41 ; 250$ & $0.44 ;-0.03 ; 51.2$ \\
\hline H2 & $\begin{array}{l}\text { Iron County, Utah, } \\
\text { USA }\end{array}$ & $\begin{array}{l}\text { Hematite } \\
\text { Quartz }\end{array}$ & $\begin{array}{l}a=503.4 ; c=1374.9 \\
a=491.4 ; c=540.7\end{array}$ & $0.42 ; 80 ; 1.15 ; 245$ & $0.41 ;-0.03 ; 51.8$ \\
\hline H3 & Elba, Italy & Hematite & $a=503.4 ; c=1375.1$ & $0.39 ; 60 ; 1.52 ; 251$ & - \\
\hline SH & $\begin{array}{l}\text { Synthetic pure } \\
\text { hematite refs }[7,9]\end{array}$ & Hematite & $a=503.5 ; c=1375.8$ & $0.28 ; 167 ; 1.24 ; 259$ & $0.41 ;-0.03 ; 51.8$ \\
\hline
\end{tabular}

The exfoliation procedure was carried out on all three specimens. Figure 2 a shows a scanning electron microscope (SEM) image of the ground precursor powder on a Si wafer. SEM images of exfoliated material on copper grids (Fig. 2b) show micron-sized hematite sheets, with little contrast. Much improved contrast is obtained in TEM, which reveals three types of structures. First are hematite nanoparticles about $100 \mathrm{~nm}$ in size (Fig 2d). Then exfoliated sheets can be seen, which are hundreds of nanometers in diameter (Fig 2c - d). In Fig 2e, HRTEM shows a lattice image, and a diffraction pattern with a (2 011$)$ zone axis. Numerous ultrafine particles < $10 \mathrm{~nm}$ in size are gathered particularly at the edges of the copper grid (Fig. 2f). EDX composition analysis on the lamellae confirms the identification of $\mathrm{Fe}_{2} \mathrm{O}_{3}$. Exfoliated sheets were found on the copper grid on multiple samples, even after repeated TEM measurements, although the sheets were estimated to represent only a minor fraction of the mass of material on the grid. Atomic force microscopy (AFM) used to determine the thickness of some of the sheets shows that their thickness ranges from $1 \mathrm{~nm}$ to more than $10 \mathrm{~nm}$ (Figures $2 \mathrm{~g}$ and $2 \mathrm{~h}$ ). 

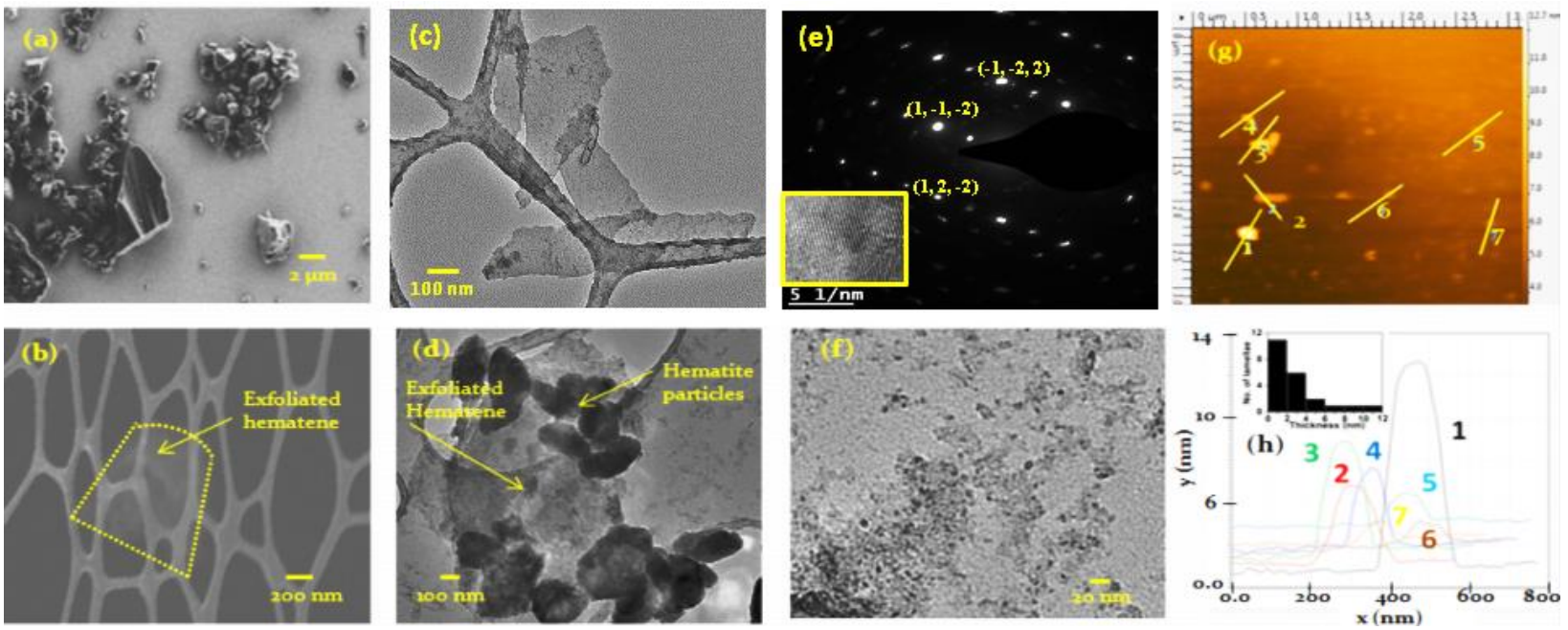

Figure 2: (a) SEM image of ground H1 powder on Si (100); (b) SEM image of a $300 \mathrm{~nm}$ exfoliated hematite sheet on a copper grid; (c) TEM image of micron-sized exfoliated hematite sheets on copper grid; (d) TEM measurements revealing hematite nanoparticles, exfoliated sheets and ultrafine nanoparticles on a copper grid; (e) SAED image of an exfoliated sheet with indexes of diffracted spots. The zone axis is (2 0 1); Inset show a magnified view of selected area under study; (f) Magnified view of ultrafine particles on copper grid; (g) AFM measurements on exfoliated sheets on Si (100) and corresponding line scans over the numbered lamellae plotted in (h) show the thicknesses in nm.

Raman spectra of exfoliated hematite show $\mathrm{A}_{1 \mathrm{~g}}$ symmetric vibration modes at 225 and $498 \mathrm{~cm}^{-1}$ and $\mathrm{E}_{\mathrm{g}}$ modes at 247, 293, 299, 412 and $613 \mathrm{~cm}^{-1}$, like those in bulk hematite, but the forbidden $E_{u}$ vibrational mode at $662 \mathrm{~cm}^{-1}$ characteristic of nanostructured materials was also observed for exfoliated hematite due to the reduced symmetry (Figure 3a). Similarly, IR spectra also show the emergence of a $\mathrm{E}_{\mathrm{u}}$ mode in the exfoliated product (Figure $\left.3 \mathrm{~b}\right)[3,11]$.
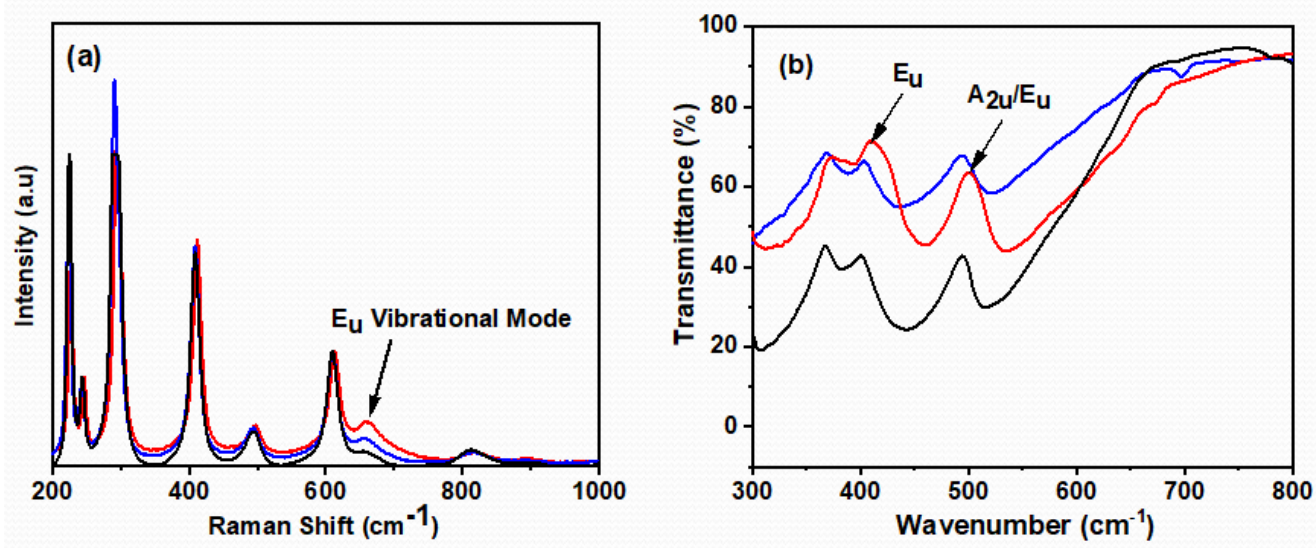
Figure 3: (a) Raman spectra of exfoliated H1 sampled from upper level of supernatant (red), the middle of the supernatant (blue) and bulk material (black); (b) shows the infra-red spectra of the same materials.
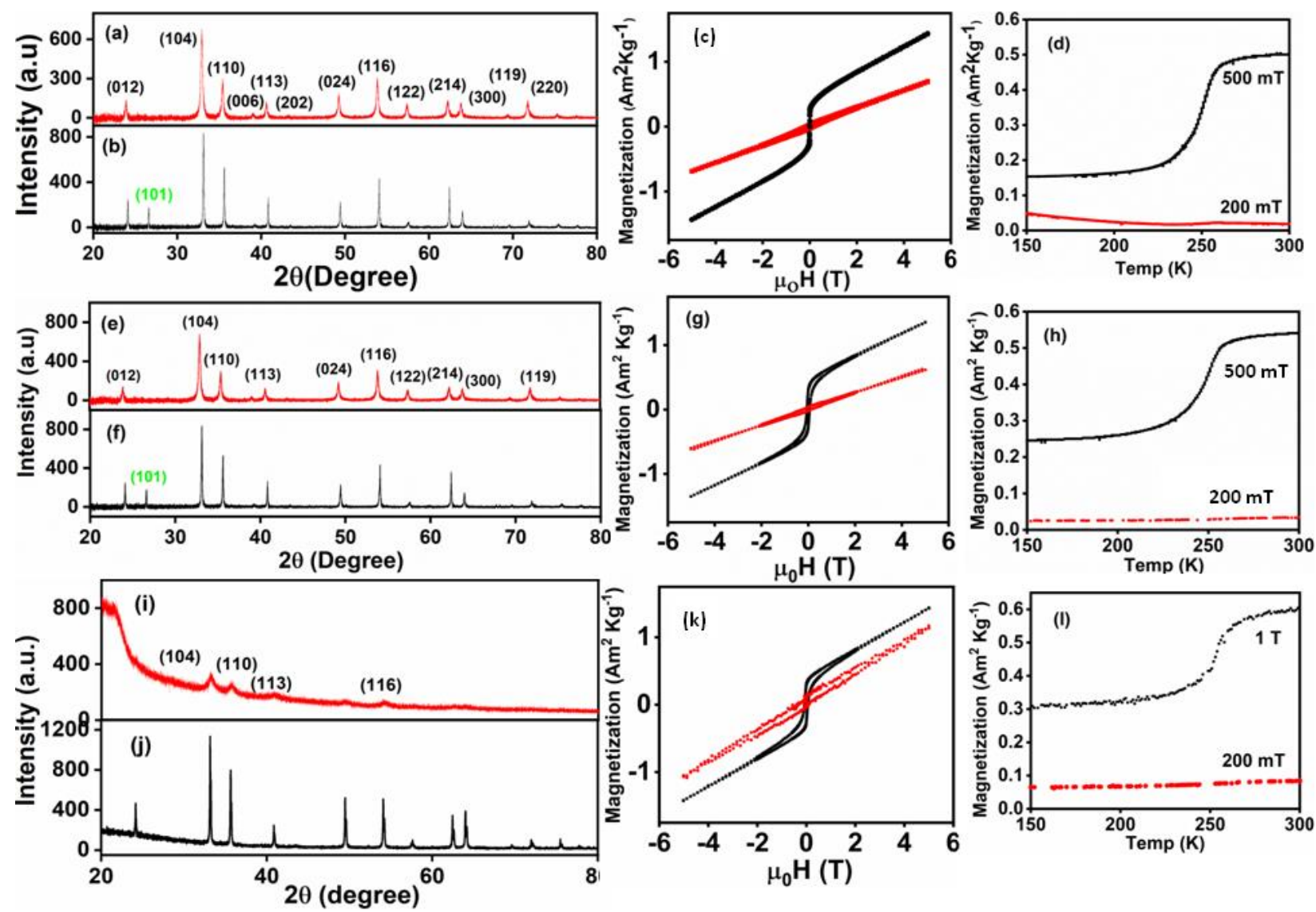

Figure 4: Left panels: XRD patterns for exfoliated (red) and bulk H1 hematite (black); Middle panels: Room-temperature hysteresis loops for exfoliated (red) and bulk H1 hematite (black); Right panels: Thermomagnetic scans for the same materials. The top row shows data for H1, the middle row shows data for $\mathrm{H} 2$ and the bottom row shows data for $\mathrm{H} 3$.

The XRD patterns of the exfoliated samples can be indexed on the hematite crystal structure with lattice parameters; $\mathrm{a}=503.1 \mathrm{pm}$ and $\mathrm{c}=1374.1 \mathrm{pm}$. No traces of any other oxide such as $a$ - or $\beta$ $\mathrm{FeOOH}, \mathrm{Fe}_{3} \mathrm{O}_{4}$ or $\gamma-\mathrm{Fe}_{2} \mathrm{O}_{3}$ were detected. Some enhancement in peak intensity for (006) and (119) compared with (104) suggests that the $c$-plane is at most a weakly-preferred exfoliated plane. No quartz reflections were observed in the XRD patterns (Figure $4 \mathrm{a}$ and $\mathrm{b}$ ). 
Antiferromagnetism in bulk hematite is attributed to $\sim 130^{\circ}$ interplane $\mathrm{Fe}^{3+}-\mathrm{O}-\mathrm{Fe}^{3+}$ antiferromagnetic superexchange and $\sim 90^{\circ}$ intraplane ferromagnetic superexchange interactions [8]. Due to the Dzyaloshinski-Moriya interaction, a small canting of moments within each sublattice results in the net ferromagnetic moment of $\sim 0.003 \mu_{\mathrm{B}}$ per $\mathrm{Fe}$ atom when the sublattice moments lie are perpendicular to $c$, but not below $T_{\mathrm{M}}$, when spins reorient to lie at an common angle of about $10^{\circ}$ to $c$, resulting in an antiferromagnetic state with an even smaller net magnetization [8]. The reason for the spin reorientation is a delicate balance of anisotropy terms of magnetocrystalline and dipolar origin, which are of opposite sign, and scale as $\left\langle S_{z}^{2}\right\rangle$ and $\left\langle S_{z}\right\rangle^{2}$ respectively [12].

Magnetization curves at room temperature and thermal scans of the magnetization in 500 $\mathrm{mT}$ from 100 to $300 \mathrm{~K}$ of bulk and exfoliated material from the upper portion of the supernatant are shown in the right-hand panels of Figure 4. There is no sign of a Morin transition in the exfoliated material, and little net magnetization is observed anywhere in this temperature range. Material sampled from the middle of the supernatant shows a magnetization in that is about half that of the bulk, and a broader Morin transition. We have repeated these experiments for all three specimens. The weak moment and the Morin transition are suppressed in each exfoliated material. There is a $8-25 \%$ reduction in the high field susceptibility of exfoliated as compared to bulk material.

Figure 5 shows typical room-temperature ${ }^{57} \mathrm{Fe}$ Mössbauer spectra of $\mathrm{H} 1$ in bulk and exfoliated form. The well-developed magnetic hyperfine pattern in Fig. 5a) is typical of bulk hematite, where the $\mathrm{Fe}^{3+}$ ions are octahedrally coordinated by oxygen. There is no trace of any paramagnetic phase. However, Mössbauer spectra of the exfoliated material show a central paramagnetic doublet that increases to $30 \%$ in relative intensity as the supernatant used to cast the absorber on filter paper is sampled closer to the surface (Fig. 5b and 5c). There is considerable uncertainty in the fit parameters of the doublet because of poor statistics arising from the very small mass of iron in the absorber. No significant difference is observed in hyperfine parameters of the magnetically ordered phase for bulk and exfoliated hematite. Table 2 summarizes yhe structural, magnetization and Mössbauer findings for the exfoliated hematite samples, which may be compared with results for the bulk materials in Table 1. The Morin transition (if it exists) is above room temperature and no evident differences are observed in the structural and magnetization analysis of exfoliated products. 


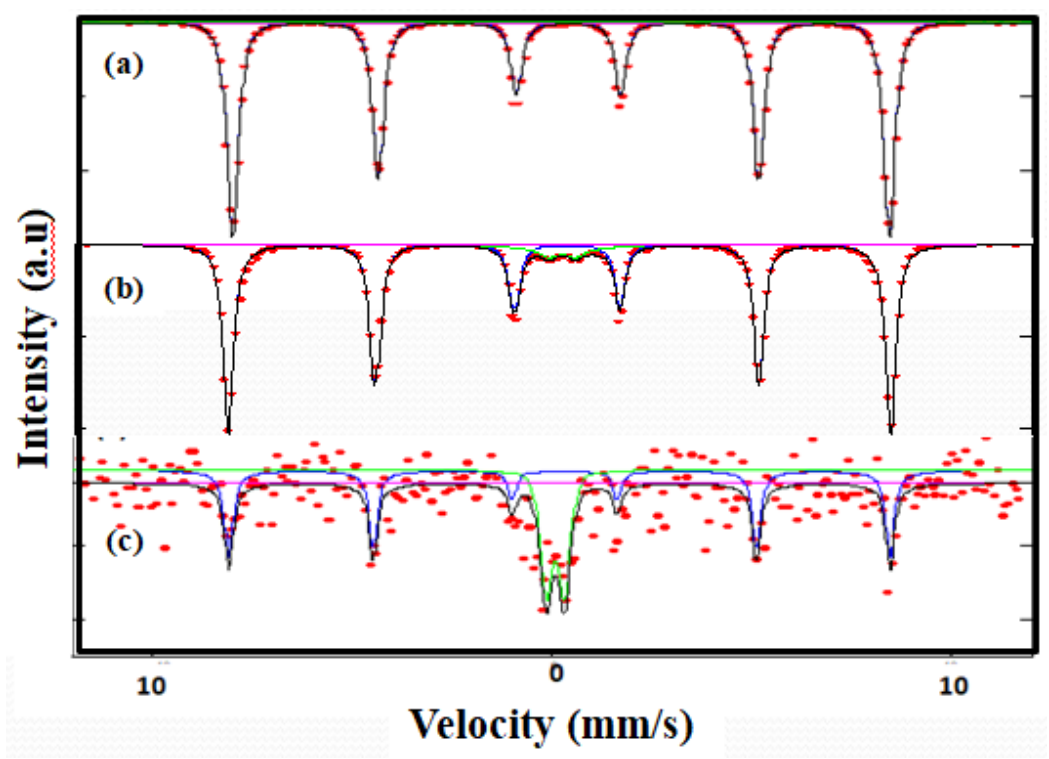

Figure 5: Room-temperature Mössbauer spectra of H1 bulk (a) and centrifuged exfoliated hematene sampled at the middle (b) and upper level (c) of the centrifuged supernatant.

Table 2: Summary of structural, magnetization and Mössbauer data for exfoliated hematites.

\begin{tabular}{|c|c|c|c|c|}
\hline Sample Name & Sample Form & $\begin{array}{l}\text { Lattice } \\
\text { parameters } \\
a, c(p m)\end{array}$ & $\begin{array}{l}{ }_{s}\left(\mathrm{Am}^{2} \mathrm{~kg}^{-1}\right) ; \\
\mu_{0} \mathrm{H}_{\mathrm{c}}(\mathrm{m} \mathrm{T}) ; \\
\chi\left(10^{-3}\right) ; T_{M}(\mathrm{~K})\end{array}$ & $\begin{array}{l}\text { Mössbauer } \\
\text { IS }\left(\mathrm{m} \mathrm{m} \mathrm{s}^{-1}\right) ; \\
\text { /QS }\left(\mathrm{m} \mathrm{m} \mathrm{s}^{-1}\right) \text {; } \\
B_{\mathrm{hf}}(\mathrm{T})\end{array}$ \\
\hline Exfoliated H1 & $\begin{array}{l}\text { Powder on filter paper } \\
\text { Upper fraction of supernatant }\end{array}$ & $\begin{array}{l}a=503.1 \\
c=1374.1\end{array}$ & $0.03 ; 130 ; 0.87 ;-$ & $\begin{array}{l}0.38 ;-0.02 ; 51.4(70 \%) \\
0.21 ; 0.40(30 \%)\end{array}$ \\
\hline Exfoliated H1 & $\begin{array}{l}\text { Powder on filter paper } \\
\text { Middle fraction of supernatant }\end{array}$ & $\begin{array}{l}a=503.3 . \\
c=1375.2\end{array}$ & $0.13 ; 72 ; 1.10 ; 214$ & $\begin{array}{l}0.40 ;-0.02 ; 51.5(97 \%) \\
0.38 ; 0.65(3 \%)\end{array}$ \\
\hline Exfoliated H2 & Powder on filter paper & $\begin{array}{l}a=504.4 \\
c=1370.4\end{array}$ & $0.04 ; 130 ; 0.94 ;-$ & $\begin{array}{l}0.41 ;-0.02 ; 51.6(95 \%) \\
0.21 ; 0.40(5 \%)\end{array}$ \\
\hline Exfoliated H3 & Powder on filter paper & $\begin{array}{l}a=503.7 \\
c=1372.5\end{array}$ & $0.10 ; 132 ; 1.27 ;-$ & - \\
\hline
\end{tabular}

\section{Discussion.}

There is substantial literature on the magnetic properties [1, 13, 14] and Mössbauer [13, 15] spectra of natural and synthetic hematite nanoparticles. It is generally found that the Morin transition moves to lower temperature with reduced particle size and that it is sharply reduced in particles smaller than about $100 \mathrm{~nm}$ [13]. Moments remain in-plane and the net weak ferromagnetism persists to the lowest temperatures. Particles smaller than about $15 \mathrm{~nm}$ in diameter exhibit superparamagnetic fluctuations at room temperature, and show a paramagnetic 
doublet as their Mössbauer spectrum. It is therefore interesting that hematite subjected to the exfoliation procedure behaves so differently; it exhibits essentially no net magnetization. The predicted ferrimagnetic structure of hematene with a net moment of the thin sheets [16] is not found in our measurements.

It is difficult to estimate quantitatively from TEM images the relative proportions of the three structures present in the supernatant namely $\sim 100 \mathrm{~nm}$ nanoparticles, ubiquitous ultrafine nanoparticles $(<10 \mathrm{~nm})$, particularly abundant in H1, and thin lamellae hundreds of $\mathrm{nm}$ in size \& 1-10 nm thick. It seems that the volumes of the three forms decrease in that order and that none of them contribute a significant net moment, since no net moment is measured. We attribute the central Mössbauer doublet to the smallest nanoparticles (approx. 15\%), which from their size may be expected to exhibit rapid magnetic relaxation at room temperature [17], while remaining antiferromagnetically coupled. If the ferric iron were paramagnetic, it would contribute noticeably to the susceptibility. In view of the high Néel temperature, we can assume the other two structures are also magnetically ordered, and produce a magnetic hyperfine spectrum that is very similar to that of bulk hematite. The thin lamellar sheets are estimated to account for only a few percent of the material on the TEM grid.

Since hematite is a planar antiferromagnet, composed of ferromagnetic $c$-plane sheets coupled antiferromagnetically [1], thin (001) lamellae would be expected to exhibit a large net magnetization $M_{\mathrm{s}} / n$ if they were composed of $n$ sheets where $n$ is odd, but zero magnetization if $n$ is even, where the sublattice magnetization $\sigma_{\mathrm{sl}} \approx 250 \mathrm{Am}^{2} \mathrm{~kg}^{-1}$. If $n$ were five on average (thickness $1.7 \mathrm{~nm}$ ) $\sigma_{\mathrm{s}}$ would be_25 $\mathrm{Am}^{2} \mathrm{~kg}^{-1}$. The measured value for the material in the upper level of the supernatant is $<0.1 \mathrm{Am}^{2} \mathrm{~kg}^{-1}$, which means that $n$ would have to be greater than 1250 , corresponding to a thickness of $430 \mathrm{~nm}$, which is obviously far too great. Both (010) and (001) sheets were considered in the original report [3]. However, from HRTEM microdiffraction patterns on exfoliated lamellae of $\mathrm{H} 1$ and $\mathrm{H} 3$, we consistently find zone axes of the sheets that are nearly perpendicular to the c-axis. The angles between the sheets and the c-axis range from 10 - 30 degrees. The $100 \mathrm{~nm}$ particles, which would normally have a Morin transition at about $200 \mathrm{~K}$, may have undergone a bulk or surface modification that weakens the D-M interaction or strengthens the uniaxial magnetocrystalline anisotropy.

The overall yield of lamellae in the exfoliation process is roughly estimated from our data to lie in the range of one part in $10^{3}$ to one part in $10^{4}$, and the yield of scarce hematene lamellae 
with a thicknesses of less than a nanometer may be a factor of 10 less, in agreement with recent modelling [18].

\section{Conclusions}

Ultrasonic exfoliation in the presence of DMF was confirmed for three natural specimens of hematite, two of them intergrown with quartz. The upper level of the supernatant after centrifuging contains mostly hematite nanoparticles; there is a minor fraction of lamellar hematite several hundreds of nanometers in diameter with thicknesses in the range $1-10 \mathrm{~nm}$. The volume fraction of material that is only a few atomic layers thick is very small. Based on these results, it would be a challenge to develop an efficient uniform exfoliation processes for oxides that could rival those for layer compounds with van der Waals interlayer bonding [19] and thereby offer a route to spintronic applications [20].

Unlike bulk hematite or hematite micro- or nano-crystals, the collections of large and small nanoparticles and lamellae of different thickness in exfoliated material are surprisingly found to possess no uncompensated moment and exhibit no Morin transition below $300 \mathrm{~K}$. This key observation is consistent with the planar antiferromagnetic structure of hematite because the cleavage is not perpendicular to (001) instead, the cleavage planes make small angle with the $c$ axis and the contributions of the two sublattices compensate over the large surface areas of the lamellae.

Acknowledgements: This project has received funding from Science Foundation Ireland through the ZEMS contract 16/IA/4534. The electron microscopy for this project was carried out at the CRANN Advanced Microscopy Laboratory at Trinity College Dublin, with support from SFI through the AMBER Centre contract 12/RC/2278. 


\section{References:}

1. Morrish, A. H. Canted Antiferromagnetism; Hematite, World Scientific, Singapore, 1994, $192 \mathrm{pp}$.

2. Jani, H. et. al., Antiferromagnetic half-skyrmyons and bimerons at room temperature Nature 59074 (2021)

3. Puthirath, A. B. et. al., Exfoliation of a non-van der Waals material from iron ore hematite. Nat. Nanotechnol. 13, 602-609 (2018).

4 Puthirath, A. B. et al., Scale-enhanced magnetism in exfoliated atomically-thick magnetite sheets, Small 16, 2004208 (2020).

5. Cheng,W. et al., Half-Unit-Cell $\alpha-\mathrm{Fe}_{2} \mathrm{O}_{3}$ Semiconductor Nanosheets with Intrinsic and Robust Ferromagnetism. J. Am. Chem. Soc. 136, 10393-10398 (2014).

6 Huang, B. et al, Layer-dependent Ferromagnetism in a van der Waals Crystal down to the Monolayer Limit, Nature 546, 270 (2017).

7 Gong, C. et. al., Two-dimensional magnetic crystals and emergent heterostructure devices. Science 363, (2019).

8. Hill, A. H. et. al., Neutron diffraction study of mesoporous and bulk hematite, $\alpha-\mathrm{Fe}_{2} \mathrm{O}_{3}$, Chem. Mater. 20, 4891 (2008).

9. $\mathrm{H} 1$ and $\mathrm{H} 2$ were supplied by Minerals Unlimited, Ridgecrest, California. H3 was supplied by Elba Minerals, Italy

10. Greenwood N. N. and Gibb. T. C., Mössbauer Spectroscopy, Chapman and Hall, London, 1972, 660 pp.

11. Jiang, J. W. et. al., Enabling unassisted solar water splitting by iron oxide and silicon, Nat. Commun. 6, 1-5 (2015).

12. Artman, J. Murphy J C and Foner, S,, Magnetic Anisotropy in Antiferromagnetic Corundum-Type Sesquioxides, Phys. Rev. 138, 912 (1965).

13. Özdemir, Ö., Dunlop, D. J. \& Berquó, T. S. Morin transition in hematite: Size dependence and thermal hysteresis. Geochemistry, Geophys. Geosystems 9, (2008).

14. Bødker, F., et. al., Magnetic properties of hematite nanoparticles. Phys. Rev. B 61, 6826 (2000). 
15. Hernandez, F. M. et al, Evidence for weak ferromagnetic moment within the basal plane of hematite natural crystals at low temperature, Geochem. Geophys. Geosyst., 14, 4444-4457 (2013).

16. Bandyopadhyay, A., et. al., Engineering Magnetic Phases in Two-Dimensional Non-van der Waals Transition-Metal Oxides. Nano Lett. 19, 7793-7800 (2019).

17. Gee, S. H., Spin Orientation of Hematite $\left(\alpha-\mathrm{Fe}_{2} \mathrm{O}_{3}\right)$ Nanoparticles During the Morin Transition, IEEE Trans. Magn., 40, 2691 (2004).

18. Padilha, A. C. M., Soares, M., Leite, E. R. \& Fazzio, A. Theoretical and Experimental Investigation of 2D Hematite. J. Phys. Chem. C 123, 16359-16365 (2019).

19. Nicolosi, V., et. al., Liquid Exfoliation of Layered Materials, Science 340, 1420 (2013).

20. Cortie, D. L. et. al., Two-Dimensional Magnets: Forgotten History and Recent Progress towards Spintronic Applications. Adv. Funct. Mater. 30, 1-15 (2020). 